\title{
Escherichia coli virulence factors iutA, an iron acquisition factor, and $i b e A$, an invasion factor, are related to severity of bacteremic acute biliary tract infections
}

\section{Mahoko Ikeda ( $\sim$ mhk-ikeda@umin.ac.jp)}

University of Tokyo Hospital: Tokyo Daigaku Igakubu Fuzoku Byoin https://orcid.org/0000-0003-47323483

\section{Tatsuya Kobayashi}

University of Tokyo Hospital: Tokyo Daigaku Igakubu Fuzoku Byoin

\section{Fumie Fujimoto}

University of Tokyo Hospital: Tokyo Daigaku Igakubu Fuzoku Byoin

\section{Yuta Okada}

University of Tokyo Hospital: Tokyo Daigaku Igakubu Fuzoku Byoin

\section{Yoshimi Higurashi}

University of Tokyo Hospital: Tokyo Daigaku Igakubu Fuzoku Byoin

\section{Keita Tatsuno}

University of Tokyo Hospital: Tokyo Daigaku Igakubu Fuzoku Byoin

\section{Shu Okugawa}

University of Tokyo Hospital: Tokyo Daigaku Igakubu Fuzoku Byoin

\section{Kyoji Moriya}

University of Tokyo Hospital: Tokyo Daigaku Igakubu Fuzoku Byoin

\section{Research}

Keywords: Extraintestinal pathogenic Escherichia coli, biliary tract infection, bacteremia, severity, virulence factors, iutA, ibeA

Posted Date: September 14th, 2020

DOI: https://doi.org/10.21203/rs.3.rs-73526/v1

License: (a) (i) This work is licensed under a Creative Commons Attribution 4.0 International License. Read Full License 


\section{Abstract}

\section{Background}

Although Escherichia coli is the most frequently isolated organism in acute biliary tract infections with bacteremia, data regarding its virulence are limited.

\section{Results}

Bacteremic acute biliary tract infection cases in a retrospective study were collected from 2013 to 2015 at a tertiary care hospital in Japan. Factors related to the severity of infection were investigated, including patient background, phylogenetic typing, and virulence factors of $E$. coli, such as adhesion, invasion, toxins, and iron acquisition.

In total, $72 \mathrm{E}$. coli strains were identified in 71 cases, most of which primarily belonged to the phylogenetic B2 group (68\%). The presence of lutA (77.3\% in the non-severe group, $46.4 \%$ in the severe group, $P=0.011)$ and $i b e A(9.1 \%$ in the non-severe group, and $35.7 \%$ in the severe group, $P=0.012$ ) were significantly associated with severity of infection, whereas patient characteristics did not relate to the severity of infection.

\section{Conclusions}

We showed that bacteremic $E$. coli strains from acute biliary tract infections belonged to a virulent (B2) group. The severity of biliary tract infection depended on iut $A$ and $i b e A$.

\section{Background}

Escherichia coli is the most frequently isolated organism in acute biliary tract infections $(1,2)$, and the associated bacteremia is mainly caused by $E$. coli (3). Biliary tract infections normally start with the stasis of bile flow. Once intestinal bacteria flow into the bile duct, an acute biliary infection can develop. The causative organisms in bile cultures of acute biliary infections were shown to be polymicrobial $(3,4)$. These pathogens were considered to be commensal with the intestinal flora whose virulence were weak.

E. coli can be differentiated depending on pathogeny. These include commensals, which are considered avirulent, intestinal pathogenic groups, extraintestinal pathogenic groups, such as uropathogenic, neonate meningitis groups, and sepsis-associated groups, and avian pathogenic $E$. coli $(5,6)$.

Acute biliary infections, such as cholangitis and cholecystitis, can develop into a severe infection with bacteremia. In cases of severe infection, mortality can reach $10 \%$ (7). This begs the question, if the causative organisms were avirulent, why did the patients suffer severe disease and even death?

We hypothesized that the causative $E$. coli were virulent, and this influenced the severity of acute biliary infection. Uropathogenic $E$. coli, which cause urinary tract infections, are well-studied, and have been 
shown to express several virulence factors, such as those involved in adhesion ( $p a p C, p a p G 2, s f a D / E$, $a f a B / C$, fimH, iha, and usp), invasion (ibeA, kpsMT2, traT, cvaC, ompT), iron acquisition (iutA, fyuA, ironEc, iucD), toxins ( $C N F 1, h l y A$, sat), and the Toll/interleukin 1 receptor (TIR) domain-containing protein (TCpC). These virulence factors play important roles at each step of infection(8). Studies on $E$. coli strains from bacteremia in biliary tract infections (BEC) are limited. A previous study showed that different prevalence of 10 virulence factors in BEC compared in E. coli strains from blood cultures of patients with acute urinary tract infections (9).

Pathogenic E. coli express many common virulence factors even at different sites of infection, but an organ-specific strategy is needed to identify specific virulence factors. These common and/or different traits are now studied rigorously to understand pathogenesis and to cope with infection at different foci.

Our study aimed to analyze the relationship between the severity of biliary tract infections and virulence of BEC strains.

\section{Results}

A total of 71 cases of bacteremic acute biliary tract infections (72 BEC isolates) were identified. We compared the severe (Pitt's score 2 or greater) group to the non-severe (score less than 2) group. Patient background, such as age, gender, and comorbidity were the same in the two groups (Table 1). More than half of the patients in both groups had abnormalities of the biliary tract. Approximately half of the patients in both groups had experienced biliary tract infections in the past. 
Table 1

Comparison of patient backgrounds and symptoms between severity of bacteremia

\begin{tabular}{|c|c|c|c|c|}
\hline & Total & $\begin{array}{l}\text { Pitt less } \\
\text { than } 2\end{array}$ & $\begin{array}{l}\text { Pitt } 2 \text { or } \\
\text { more }\end{array}$ & $\begin{array}{l}P \\
\text { value }\end{array}$ \\
\hline numbers & 71 & 44 & 27 & \\
\hline age (median, range) & $\begin{array}{l}75(31- \\
94)\end{array}$ & $\begin{array}{l}72.9(34- \\
93)\end{array}$ & $\begin{array}{l}72(31- \\
94)\end{array}$ & 0.38 \\
\hline gender (male: female) & F28 M43 & F16 M28 & F12 M15 & 0.618 \\
\hline nursing home & 4 & 2 & 2 & 0.632 \\
\hline antibiotic use within 3 months & 29 & 19 & 10 & 0.479 \\
\hline Charlson index (median, range) & $4(0-11)$ & $4(0-11)$ & $3(0-8)$ & 0.104 \\
\hline collagen diseases & 4 & 4 & 0 & 0.29 \\
\hline diabetes mellitus without organ involvement & 7 & 3 & 4 & 0.415 \\
\hline diabetes mellitus with organ involvement & 20 & 17 & 3 & 0.015 \\
\hline malignancy & 49 & 31 & 18 & 0.613 \\
\hline abnormality of biliary tract & 43 & $29(65.9 \%)$ & $14(52 \%)$ & 0.318 \\
\hline abnormality of gastrointestinal tract & 38 & 25 & 13 & 0.625 \\
\hline previous history of acute biliary infection & 33 & 21 & 12 & 0.811 \\
\hline previous history of bacteremia & 11 & 7 & 4 & 1 \\
\hline $\begin{array}{l}\text { duration between onset of symptoms and } \\
\text { admission (days) }\end{array}$ & $\begin{array}{l}0(-24- \\
10)\end{array}$ & $0(-24-4)$ & $0(-10-10)$ & 0.924 \\
\hline fever & 39 & 23 & 16 & 0.629 \\
\hline immunosuppressant use & 27 & 19 & 8 & 0.318 \\
\hline artificial device in biliary tract & 19 & 11 & 8 & 0.784 \\
\hline
\end{tabular}

Table 2 shows the comparison of laboratory data by severity. Liver enzymes and biliary markers were elevated to the same degree except for alkaline phosphatase. Median white blood cell counts were higher in the non-severe group than in the severe group. 
Table 2

Comparison of laboratory data and severity of bacteremia

\begin{tabular}{|c|c|c|c|c|}
\hline & total & Pitt less than 2 & Pitt 2 or more & $\begin{array}{l}P \\
\text { value }\end{array}$ \\
\hline $\begin{array}{l}\text { white blood cell counts (per } \mu \mathrm{L} \text { ) } \\
\text { median (min-max) }\end{array}$ & $\begin{array}{l}9800(1200- \\
25600)\end{array}$ & $\begin{array}{l}10500(2400- \\
25600)\end{array}$ & $\begin{array}{l}8600(1200- \\
23200)\end{array}$ & 0.0492 \\
\hline platelets $(\times 10000 / \mu \mathrm{L})$ & $\begin{array}{l}16.1(7.0- \\
38.1)\end{array}$ & $17.6(7-38.1)$ & $\begin{array}{l}15.2(7.0- \\
35.1)\end{array}$ & 0.211 \\
\hline Albumin (g/dL) & $3.2(1.0-4.5)$ & $3.15(1.0-4.5)$ & $3.5(2.0-4.2)$ & 0.136 \\
\hline total bilirubin (mg/dL) & $2.0(0.4-11.6)$ & $2.0(0.4-11.6)$ & $2.2(0.5-7.0)$ & 0.148 \\
\hline AST (U/L) & $\begin{array}{l}100(16- \\
1591)\end{array}$ & $98(16-1026)$ & $\begin{array}{l}133(17- \\
1591)\end{array}$ & 0.152 \\
\hline ALT (U/L) & $91(11-724)$ & $77(13-569)$ & $100(11-724)$ & 0.4 \\
\hline g-GTP (U/L) & $\begin{array}{l}328(11- \\
2118)\end{array}$ & $346(11-2118)$ & $288(14-817)$ & 0.621 \\
\hline ALP (U/L) & $\begin{array}{l}733(143- \\
3520)\end{array}$ & $\begin{array}{l}855(143- \\
3520)\end{array}$ & $\begin{array}{l}594(232- \\
3278)\end{array}$ & 0.026 \\
\hline CRP (mg/dL) & $\begin{array}{l}3.76(0.08- \\
22.32)\end{array}$ & $\begin{array}{l}3.48(0.08- \\
18.53)\end{array}$ & $\begin{array}{l}5.0(0.11- \\
22.32)\end{array}$ & 0.687 \\
\hline
\end{tabular}

Next, we compared the phylogenetic groups and virulence factors of BEC by severity (Table 3). BEC isolates mainly belonged to the phylogenetic group B2 (68\%). ST 131 (22.2\%) and ST 95 (19.4\%) were detected frequently between four STs by multi-locus sequencing typing (MLST), but more than half belonged to other STs. The most detected phylogenetic group was B2 in both groups $(70.5 \%$ in nonsevere, $64.3 \%$ in severe), but the proportion of each phylogenetic group was similar. 
Table 3

Comparison of patterns of phylogenetic groups and prevalence of virulence factors between non-severe and severe groups.

\begin{tabular}{|c|c|c|c|c|}
\hline & Total & Pitt less than 2 & Pitt 2 or more & $P$ value \\
\hline phylogenetic group & 72 & 44 & 28 & 0.732 \\
\hline A & 4 & 2 & 2 & \\
\hline B1 & 8 & 3 & 5 & \\
\hline B2 & 49 & 31 & 18 & \\
\hline D & 1 & 1 & 0 & \\
\hline$E$ & 6 & 4 & 2 & \\
\hline$F$ & 4 & 3 & 1 & \\
\hline MLST & & & & 0.359 \\
\hline ST131 & 17 & 13 & 4 & \\
\hline ST95 & 14 & 8 & 6 & \\
\hline ST73 & 5 & 4 & 1 & \\
\hline ST69 & 1 & 1 & 0 & \\
\hline others & 35 & 18 & 17 & \\
\hline \multicolumn{5}{|l|}{ virulence factors } \\
\hline papC & 21 & 15 & 6 & 0.296 \\
\hline$s f a D / E$ & 8 & 4 & 4 & 0.703 \\
\hline CNF1 & 9 & 5 & 4 & 0.728 \\
\hline$i u c D$ & 24 & 18 & 6 & 0.124 \\
\hline$a f a B / C$ & 2 & 1 & 1 & 1 \\
\hline h/yA & 9 & 5 & 4 & 0.728 \\
\hline fyuA & 52 & 35 & 17 & 0.108 \\
\hline cvaC & 6 & 6 & 0 & 0.753 \\
\hline $\mathrm{fimH}$ & 69 & 42 & 27 & 1 \\
\hline iutA & 47 & 34 & 13 & 0.011 \\
\hline$i b e A$ & 14 & 4 & 10 & 0.012 \\
\hline iha & 23 & 16 & 7 & 0.438 \\
\hline
\end{tabular}




\begin{tabular}{|lllll|}
\hline & Total & Pitt less than 2 & Pitt 2 or more & P value \\
\hline ompT & 6 & 6 & 0 & 0.075 \\
\hline kpsMT2 & 51 & 33 & 18 & 0.427 \\
\hline papG2 & 10 & 8 & 2 & 0.297 \\
\hline usp & 56 & 35 & 21 & 0.773 \\
\hline ironEC & 16 & 10 & 6 & 1 \\
\hline sat & 22 & 16 & 6 & 0.202 \\
traT & 49 & 33 & 16 & 0.128 \\
\hline TcpC & 12 & 9 & 3 & 0.346 \\
\hline
\end{tabular}

The presence of iutA (77.3\% in the non-severe group, $46.4 \%$ in the severe group, $P=0.011)$ and $i b e A(9.1 \%$ in non-severe group, and $35.7 \%$ in severe group, $P=0.012$ ) were significantly associated with severity, whereas patient characteristics were not related to severity.

\section{Discussion}

In this study, BEC mainly belonged to group B2, which is known as a virulent group, and ST131. Although acute biliary tract infections are caused by obstruction/stasis of biliary flow and influx of commensal bacteria into the biliary tract, BEC detected from blood cultures belonged to a pathogenic group.

The human intestinal tract has been recognized as a reservoir of extraintestinal pathogenic $E$. coli strains, such as uropathogenic E. coli (10). BEC may also hide in the gut. Once BEC translocates into the biliary tract due to stasis/obstruction, acute biliary tract infections can occur and develop into bacteremia.

Commensal E. colistrains, once considered as avirulent, are now known to express many of the same virulence factors, such as adhesion factors, as pathogenic $E$. coli strains. This is because these factors are needed for persistent colonization in the gut. E. coli has resistance against the effects of bile (11). In response to bile stress, both commensal and pathogenic $E$. coli strains, and especially enteropathogenic E. coli strains, activate stress response pathways $(12,13)$, efflux pumps $(14)$, and production of toxins (15) in the gut. As there are high concentrations of bile acid in the biliary tract, resistance against bile might play an important role in pathogenicity.

In our study, other virulence factors which are needed during infection, such as iron acquisition, adhesion, and invasion, were analyzed. Factors needed for colonization of the gut as compared to those needed during biliary tract infection must be analyzed to clarify specific virulence factors of the biliary tractpathogenic $E$. coli strains. The differences between commensal and enteropathogenic $E$. coli strains in the gut have yet to be clarified. 
In the present study, iutA was found at a lower level in the severe group than in the non-severe group. lutA codes for the aerobactin siderophore ferric receptor protein, which has a role in facilitating iron acquisition by mediating the uptake of siderophores (16). In a chicken infection model, iutA expression in extraintestinal pathogenic E. coli strains was at least 50 -fold higher in organ tissues compared to in vitro grown bacteria during infection (17). In mammalian hosts, iron is tightly bound to various proteins, such as hemoproteins and ferritin (18), making free iron for use by pathogenic bacteria scarce. In biliary tract infections, bile is an iron-limiting environment (19). Bile stress also causes increased mRNA levels for virulence genes associated with iron scavenging in $E$. coli (20). Therefore, E. colistrains harboring the iutA gene may become competitive in bile, but those without the iutA gene might find it difficult to proliferate in bile and escape into the blood. An iutA vaccine protected mice in a sepsis challenge model (21) and UTI model (22). Because iutA as an antigen might be easily recognized by host immune systems, $E$. coli strains harboring the iutA gene may be easy to eliminate in bile.

In contrast, $E$. coli strains containing the $i b e A$ gene were found in a higher proportion in the severe group. IbeA codes for a $50 \mathrm{kDa}$ protein used for penetration of human brain microvascular endothelial cells to invade through the blood brain barrier, and is thought to have an important role in neonatal meningitis $(23,24)$. IbeA may also be essential for invasion into intestinal epithelial cells and macrophages (25). No information regarding the relationship between $i b e A$ and biliary tract epithelium cells has been reported; therefore, the mechanism of $i b e A$ on the severity of acute biliary infection is unknown. ibeA inhibitors have been discovered to prevent invasion of human brain microvascular endothelial cells in vitro (26). It would be worthwhile to investigate whether $E$. coli strains harboring ibe $A$ can perform internalization, and whether $i b e A$ is essential to invade from bile duct to vessels through in vitro experiments using ibeA inhibitors.

In a rat model with common bile duct ligation, clearance of $E$. coli from blood and trapping rates in the liver were decreased compared to controls, and also showed decreased phagocytic activity and superoxide production of Kupffer cells (27). In our results, no significant relation was observed between severity of infection and patient background, such as abnormalities in the biliary tract and laboratory data of jaundice markers, except ALP.

This study has several limitations. First, although cholangitis is a polymicrobial infection (3), we investigated only one $E$. coli strain from each patient without analyzing other $E$. coli strains found in the bile. This comparison might reveal whether ibeA-positive strains can more easily escape or invade into the blood compared to strains that remained in the bile. Second, this study was conducted at a single institution. A larger multi-center study is needed to assess potential bias in the epidemiology of phylogenetic patterns and virulence factors.

\section{Conclusion}

We showed that many BEC belonged to a virulent group (B2) with a high prevalence of ST131. The severity of biliary tract infections depended on the presence of $i u t A$ and $i b e A$ in BEC. 


\section{Methods}

\section{Patients}

This retrospective study was conducted at the University of Tokyo Hospital, a 1217-bed tertiary-care teaching hospital in Tokyo, Japan. Patients with acute biliary tract infection who also had E. coli isolates detected in their blood were included in the study from April 2013 to February 2015. Each patient in this study was included only once, even if the patients repeatedly suffered $E$. coli bacteremia with acute biliary tract infection. Patient data, including clinical symptoms and microbiological data were collected from the medical records.

\section{Data collection and definitions}

Patient data collected included age, sex, underlying disease (diabetes mellitus, malignancy with or without metastasis, lymphoma, and collagen disease), use of immunosuppressants, biliary tract abnormalities, such as insertion of an intrabiliary stent or surgery for biliary carcinoma, gastrointestinal tract abnormalities, and past history of acute biliary infection and bacteremia. History of residence in a nursing home and antibiotic use within three months before onset of bacteremia was also noted. Collected patient laboratory data included white blood cell counts, platelet counts, total bilirubin, aspartate aminotransferase, alanine aminotransferase, alkaline phosphatase, gamma glutamyltransferase, and C-reactive protein. Cases in which $E$. coli was detected from blood cultures obtained within $48 \mathrm{~h}$ after admission were defined as community acquired infection. Others were considered hospital acquired infections.

Biliary tract infection was defined according to the Tokyo guideline (28). Cholangitis was defined in cases where all the following sings were positive: 1 ) generalized inflammation sign such as fever (more than $38.0^{\circ} \mathrm{C}$ ), elevation of inflammation indicators in blood tests (white blood cell counts less than $4000 / \mathrm{mL}$ or more than $10,000 / \mathrm{mL}$, and C-reactive protein $1 \mathrm{mg} / \mathrm{dL}$ or more), 2) signs of bile stasis such as jaundice (total bilirubin $2 \mathrm{mg} / \mathrm{dL}$ or more), elevation of liver function and biliary function tests (more than one-anda-half times of the upper limit for normal values for alkaline phosphatase(ALP), gamma-

glutamyltransferase(g-GTP), aspartate aminotransferase(AST), or alanine aminotransferase(ALT), normal range; ALP 106-322 U/L, g-GTP 13-64 U/L in men and 9-32 U/L in women, AST 13-30 U/L, ALT 10-42 U/L in men and 7-23 $\mathrm{U} / \mathrm{L}$ in women, respectively), and 3) imaging of biliary tract abnormalities, such as dilatation of the biliary tract or the presence of a stent, and constriction. Cholecystitis was defined as: 1) localized clinical signs such as Murphy's sign and pain in right upper abdomen, 2) generalized inflammation such as fever and elevation of inflammation indicators in blood tests, and 3) typical findings, such as acute cholecystitis with echocardiography or CT scan. Severity was divided using the Pitt bacteremia score (29) as severe (score of 2 or more) and non-severe (score less than 2).

\section{Microbiological procedures}


All isolates were identified using the Walkaway system (Siemens, Berlin, Germany) or matrix-assisted laser desorption/ionization time-of-flight mass spectrometry (using the MALDI Biotyper; Bruker Daltonik, Germany).

Assignment of $E$. coli isolates to phylogenetic groups, such as A, B1, B2, C, D, E, and F, was determined by the quadruplex polymerase chain reaction (PCR) method as described by Clermont et al (30). For rapid identification of $E$. coli sequence types (STs) $69,73,95$, and 131, the multilocus sequence typing PCR method (31) was performed. The prevalence of 20 virulence factors ( $p a p C, s f a D / E, C N F 1$, iucD, afaB/C, hlyA, tcpC, fyuA, cvaC, fimH, iutA, ibeA, iha, ompT, kpsMT2, papG2, usp, ironEc, sat, and traT) were screened by multiplex PCR using extracted $E$. coli genomic DNA according to previous reports (32-38).

\section{Statistical analysis}

The two-tailed Fisher's exact test was used for analysis of categorical data. Non-parametric data were analyzed using the Mann-Whitney $U$ test. Values of $P<0.05$ were considered significant. All statistical analyses were performed using JMP Pro version 11 software (SAS Institute, Cary, NC, USA).

\section{Ethical considerations}

This study was approved by the research ethics committee at the University of Tokyo Hospital. Obtaining written informed consent from each patient was waived because it was an observational retrospective study. The data were analyzed anonymously.

\section{Declarations}

\section{Ethics approval and consent to participate}

This study was approved by the research ethics committee at the University of Tokyo Hospital. Obtaining written informed consent from each patient was waived because it was an observational retrospective study. The data were analyzed anonymously.

\section{Consent for publication}

Not applicable.

\section{Availability of data and materials}

The datasets used and/or analyzed during the current study are available from the corresponding author on reasonable request.

\section{Competing interests}

The authors declare that they have no competing interests. 


\section{Funding}

This work was supported by JSPS KAKENHI Grant Number JP18K16171. The funding agencies did not have a role in the design of the study or the collection, analysis, and interpretation of data.

\section{Authors' contributions}

MI designed this study, acquired and analyzed the patients' data, and was a major contributor in writing the manuscript. MI and TK performed PCR to analyze virulence of E. coli. FF and TH identified and collected the E. coli strains. KT made database of bacteremia, $\mathrm{YO}, \mathrm{SO}$ and $\mathrm{KM}$ revised the manuscript. All authors read and approved the final manuscript.

\section{Acknowledgments}

Not applicable

\section{References}

1. Gu XX, Zhang MP, Zhao YF, Huang GM. Clinical and microbiological characteristics of patients with biliary disease. World J Gastroenterol. 2020;26(14):1638-46.

2. Kruis T, Guse-Jaschuck S, Siegmund B, Adam T, Epple HJ. Use of microbiological and patient data for choice of empirical antibiotic therapy in acute cholangitis. BMC Gastroenterol. 2020;20(1):65.

3. Weber A, Schneider J, Wagenpfeil S, Winkle P, Riedel J, Wantia N, et al. Spectrum of pathogens in acute cholangitis in patients with and without biliary endoprosthesis. J Infect. 2013;67(2):111-21.

4. Rerknimitr R, Fogel EL, Kalayci C, Esber E, Lehman GA, Sherman S. Microbiology of bile in patients with cholangitis or cholestasis with and without plastic biliary endoprosthesis. Gastrointest Endosc. 2002;56(6):885-9.

5. Sarowska J, Futoma-Koloch B, Jama-Kmiecik A, Frej-Madrzak M, Ksiazczyk M, Bugla-Ploskonska G, et al. Virulence factors, prevalence and potential transmission of extraintestinal pathogenic Escherichia coli isolated from different sources: recent reports. Gut Pathog. 2019;11:10.

6. Croxen MA, Finlay BB. Molecular mechanisms of Escherichia coli pathogenicity. Nat Rev Microbiol. 2010;8(1):26-38.

7. Lee JG. Diagnosis and management of acute cholangitis. Nat Rev Gastroenterol Hepatol. 2009;6(9):533-41.

8. Flores-Mireles AL, Walker JN, Caparon M, Hultgren SJ. Urinary tract infections: epidemiology, mechanisms of infection and treatment options. Nat Rev Microbiol. 2015;13(5):269-84.

9. Wang MC, Tseng CC, Chen CY, Wu JJ, Huang JJ. The role of bacterial virulence and host factors in patients with Escherichia coli bacteremia who have acute cholangitis or upper urinary tract infection. 
Clin Infect Dis. 2002;35(10):1161-6.

10. Manges AR, Johnson JR. Reservoirs of Extraintestinal Pathogenic Escherichia coli. Microbiol Spectr. 2015;3(5).

11. Begley M, Gahan CG, Hill C. The interaction between bacteria and bile. FEMS Microbiol Rev. 2005;29(4):625-51.

12. Joffre E, Nicklasson M, Alvarez-Carretero S, Xiao X, Sun L, Nookaew I, et al. The bile salt glycocholate induces global changes in gene and protein expression and activates virulence in enterotoxigenic Escherichia coli. Sci Rep. 2019;9(1):108.

13. Rodriguez-Beltran J, Rodriguez-Rojas A, Guelfo JR, Couce A, Blazquez J. The Escherichia coli SOS gene dinF protects against oxidative stress and bile salts. PLoS One. 2012;7(4):e34791.

14. Rosenberg EY, Bertenthal D, Nilles ML, Bertrand KP, Nikaido H. Bile salts and fatty acids induce the expression of Escherichia coli AcrAB multidrug efflux pump through their interaction with Rob regulatory protein. Mol Microbiol. 2003;48(6):1609-19.

15. Bustamante P, Vidal R. Repertoire and Diversity of Toxin - Antitoxin Systems of Crohn's DiseaseAssociated Adherent-Invasive Escherichia coli. New Insight of T his Emergent E. coli Pathotype. Front Microbiol. 2020;11:807.

16. Robinson AE, Heffernan JR, Henderson JP. The iron hand of uropathogenic Escherichia coli: the role of transition metal control in virulence. Future Microbiol. 2018;13:745-56.

17. Chouikha I, Bree A, Moulin-Schouleur M, Gilot P, Germon P. Differential expression of iutA and ibeA in the early stages of infection by extra-intestinal pathogenic E. coli. Microbes Infect. 2008;10(4):4328.

18. Miethke M, Marahiel MA. Siderophore-based iron acquisition and pathogen control. Microbiol Mol Biol Rev. 2007;71(3):413-51.

19. Urdaneta V, Casadesus J. Interactions between Bacteria and Bile Salts in the Gastrointestinal and Hepatobiliary Tracts. Front Med (Lausanne). 2017;4:163.

20. Hamner S, Mclnnerney K, Williamson K, Franklin MJ, Ford TE. Bile salts affect expression of Escherichia coli 0157:H7 genes for virulence and iron acquisition, and promote growth under iron limiting conditions. PLoS One. 2013;8(9):e74647.

21. Mellata M, Mitchell NM, Schodel F, Curtiss RR, Pier GB. Novel vaccine antigen combinations elicit protective immune responses against Escherichia coli sepsis. Vaccine. 2016;34(5):656-62.

22. Forsyth VS, HimpsI SD, Smith SN, Sarkissian CA, Mike LA, Stocki JA, et al. Optimization of an Experimental Vaccine To Prevent Escherichia coli Urinary Tract Infection. mBio. 2020;11(2).

23. Huang SH, Wan ZS, Chen YH, Jong AY, Kim KS. Further characterization of Escherichia coli brain microvascular endothelial cell invasion gene ibeA by deletion, complementation, and protein expression. J Infect Dis. 2001;183(7):1071-8.

24. Huang SH, Chen YH, Fu Q, Stins M, Wang Y, Wass C, et al. Identification and characterization of an Escherichia coli invasion gene locus, ibeB, required for penetration of brain microvascular endothelial 
cells. Infect Immun. 1999;67(5):2103-9.

25. Cieza RJ, Hu J, Ross BN, Sbrana E, Torres AG. The IbeA invasin of adherent-invasive Escherichia coli mediates interaction with intestinal epithelia and macrophages. Infect Immun. 2015;83(5):1904-18.

26. Xu X, Zhang L, Cai Y, Liu D, Shang Z, Ren Q, et al. Inhibitor discovery for the E. coli meningitis virulence factor IbeA from homology modeling and virtual screening. J Comput Aided Mol Des. 2020;34(1):11-25.

27. Tomioka M, linuma H, Okinaga K. Impaired Kupffer cell function and effect of immunotherapy in obstructive jaundice. J Surg Res. 2000;92(2):276-82.

28. Yokoe M, Hata J, Takada T, Strasberg SM, Asbun HJ, Wakabayashi G, et al. Tokyo Guidelines 2018: diagnostic criteria and severity grading of acute cholecystitis (with videos). J Hepatobiliary Pancreat Sci. 2018;25(1):41-54.

29. Paterson DL, Ko WC, Von Gottberg A, Mohapatra S, Casellas JM, Goossens H, et al. International prospective study of Klebsiella pneumoniae bacteremia: implications of extended-spectrum betalactamase production in nosocomial Infections. Ann Intern Med. 2004;140(1):26-32.

30. Clermont O, Christenson JK, Denamur E, Gordon DM. The Clermont Escherichia coli phylo-typing method revisited: improvement of specificity and detection of new phylo-groups. Environ Microbiol Rep. 2013;5(1):58-65.

31. Doumith M, Day M, Ciesielczuk H, Hope R, Underwood A, Reynolds R, et al. Rapid identification of major Escherichia coli sequence types causing urinary tract and bloodstream infections. J Clin Microbiol. 2015;53(1):160-6.

32. Johnson JR, Brown JJ. A novel multiply primed polymerase chain reaction assay for identification of variant papG genes encoding the Gal(alpha 1-4)Gal-binding PapG adhesins of Escherichia coli. J Infect Dis. 1996;173(4):920-6.

33. Johnson JR, Stell AL. Extended virulence genotypes of Escherichia coli strains from patients with urosepsis in relation to phylogeny and host compromise. J Infect Dis. 2000;181(1):261-72.

34. Nakano M, Yamamoto S, Terai A, Ogawa O, Makino SI, Hayashi H, et al. Structural and sequence diversity of the pathogenicity island of uropathogenic Escherichia coli which encodes the USP protein. FEMS Microbiol Lett. 2001;205(1):71-6.

35. Ananias M, Yano T. Serogroups and virulence genotypes of Escherichia coli isolated from patients with sepsis. Braz J Med Biol Res. 2008;41(10):877-83.

36. Cirl C, Wieser A, Yadav M, Duerr S, Schubert S, Fischer H, et al. Subversion of Toll-like receptor signaling by a unique family of bacterial Toll/interleukin- 1 receptor domain-containing proteins. Nat Med. 2008;14(4):399-406.

37. Rodriguez-Bano J, Mingorance J, Fernandez-Romero N, Serrano L, Lopez-Cerero L, Pascual A, et al. Virulence profiles of bacteremic extended-spectrum beta-lactamase-producing Escherichia coli: association with epidemiological and clinical features. PLoS One. 2012;7(9):e44238.

38. Dissanayake DR, Octavia S, Lan R. Population structure and virulence content of avian pathogenic Escherichia coli isolated from outbreaks in Sri Lanka. Vet Microbiol. 2014;168(2-4):403-12. 